Interesse, daß sich in dem Jodvorkommen eine Differenzierung zeigt, je nach Art des Salzminerals. In dem Carnallit von Kalusz hat sich kein Jod nachweisen lassen, im Kainit derselben Lagerstätte $8,7 \mathrm{mg}$ in $10 \mathrm{~kg}$; in dem Carnallit von Neustaßfurt ist selbst in $100 \mathrm{~kg}$ kein Jod auffindbar, während es sich im Sylvin des Hartsalzes anreichert.

Für das Vorkommen des Ammoniaks haben Biltz und Marcus 21) eine ähnliche Differenzierung festgestelit. Sie ist, da der Ammoniakgehalt dem Carnallitgehalt parallel läuft, leicht zu erklären durch die Annahme, daß das Ammoniak in Form von A m moniumearnallit vorliegt, welcher mit gewöhnlichem Carnallit Mischkrystalle bildet.

Weniger leicht verständlich ist die verschiedenartige Verbreitung des $\mathrm{J}$ od s. Jodcarnallit bildet, wie $B$ o e k e nachgewiesen hat, mit gewöhnlichem Carnallit keine Mischkrystalle, und damit steht in guter Ubereinstimmung, daß gerade im Carnallit kein Jod zu finden ist. Wenn sich aber im galizischen Kainit das Jod anreichert, wenn es im deutschen Sylvin und in ganz minimaler Menge auch im jüngeren Steinsalze nachweisbar ist, so müssen zu diesen Salzen die betreffenden Jodide doch wohl eine gewisse Affinität, chemischer oder physikalischer Art, besitzen.

Es sei schließlich auf $\mathrm{zwei}$ Schlußfolgerungen - eine spezielle und eine allgemeinere - hingewiesen, welche sich aus den festgestellten Tatsachen für die Bild u n g der Salzlager ziehen lassen.

Die speziellere Folgerung betrifft die Bildung des Hartsalzes von Bleicherode und von Neu-Staßfurt. Hartsalz wird vielfach noch als ein Umwandlungsprodukt betrachtet, welches durch Auswaschung kieserithaltigen Carnallits entstanden sei. Wenn aber das Hartsalz, wie nachgewiesen wurde, jod hal $\mathrm{t} \mathrm{g}$ ist und der Carnallit jodfrei, dann ist eine Bildung dieses Hartsalzes aus Carnallit meines Erachtens so gut wie ausgeschlossen. Vielmehr scheint mir in dieser Feststellung ein zwingender Grund für die Notwendigkeit zu liegen, eine p r i m är e Ausscheidung des Hartsalzes aus einer etwas Jod enthaltenden Mutterlauge anzunehmen, wie auch der Jodgehalt des im Haselgebirge-Salzton eingelagerten Kainits von Kalusz für seine primäre Natur spricht.

Die zweite Schlußfolgerung bezieht sich auf die Bildung der deutschen Sal7lager im allgemeinen. Der quantitativ ermittelte Jodgehalt ist ein ä u Berst geringer, sehr viel geringer als der Jodgehalt, welchen die beim Eindunsten von Mérwasser auskrystallisierenden Salze aufweisen. Einem Jodgehalt von $0,83 \mathrm{mg}$ in $10 \mathrm{~kg}$ Seesalz von Berre steht ein Gehalt von $0,075 \mathrm{mg}$ im jüngeren Steinsalz gegenüber, einem Gehalt von mehr als $1,03 \mathrm{mg}$ Jod in $10 \mathrm{~kg}$,Sels mixtes" ein Gehalt von $0,1 \mathrm{mg}$ in $10 \mathrm{~kg}$ Hartsalz von Bleicherode, dabei enthalten dio "Sels mixtes“ noch gar kein Chlorkalium, welches besonders geeignet scheint, Jod zu binden.

Im Kainit von Kalusz, der wahrscheinlich direkt aus dem Meerwasser abgesetzt ist, findet sich der mehr als 20 facho Jodgehalt wie in dem jodreichsten deutschen Salzmineral, dem reinen Sylvin.

21) Z. anorg. Chem. 62, 184 (1909). Diese Z. 22, 1371 (1908).
Diese Tatsachen stehen in Ubereinstimmung mit der $W$ a $l$ t h e r schen Anschauung von der Bildung der Zechsteinsalze, eine Anschauung, die ich auf dem 4. deutschen Kalitage in Nordhausen durch gewichtige und für mich wenigstens entscheidende chemische Gründe zu stützen gesucht habe.

Denn wenn ein ursprünglich gewaltige Flächen bedeckendes Zechsteinmeer als Binnensee allmählich im Wüstenklima verdunstete und mehr und mehr einschrumpfte, dann war es unausbleiblich, daß ein großer Teil auch der leicht löslichen Salze in Lagunen eintrocknete, dann später wieder durch atmosphärische Niederschläge gelöst und schlieBlich den an den tiefsten Stellen sich bildenden Becken zugeführt wurde. Das aber sind Bedingungen, welche eine Zersetzung des Jodmagnesiums unter den Strahlen der Wüstensonne und die Wegführung eines großen Teiles des Jods durch den Wind veranlassen mußten.

\section{Die Entwicklungsgeschichte des Hegelerschen Blende-Röstofens und sein Verbreitungsgebiet.}

Von Hütteninspektor Dr. Oтto Mürhhaeuser. (Eingeg. 12.11. 1910.)

Der mechanische Röstofen der $\mathrm{M}$ a $\mathrm{t} \mathrm{t}$ h i e $\mathrm{B}$ en und $H$ egeler $Z$ in c Co. in La Salle dient ausschließlich zum Rösten der Zinkblende. Das Abrösten derselben ist jedoch weit schwieriger als beim Schwefelkies, weil die Zinkblende selten mehr als $30 \%$ Schwefel, häufig weniger, oft nur die Hälfte davon enthält. Andererseits ist sie auch an und für sich weniger leicht zu entschwefeln, und unter Umständen bildet sich das schwer zerlegbare Zinksulfat. Da aber für die Herstellung von Zink der Schwefel möglichst vollständig abgeröstet werden muB, und dio für die Pyritabröstung konstruierten Öfen für Blende nicht verwendbar sind, so mußte früher die Blende ausschließlich in Flammöfen mit direkter Kohlenfeuerung totgeröstet werden, wobei alle Schwefligsäure zum Nachteil der Pflanzenwelt in die Atmosphäre entwich. Dieser Zustand würde vielleicht noch länger angedauert haben, und, wie heute noch in Amerika, auch in Europa dort, wo kein lohnender Absatz für Schwefelsäure vorhanden ist, weiter bestehen, wenn nicht der durch den sauren Hüttenrauch $^{1}$ ) angerichtete Schaden die Behörden zunächst in England, dann auch auf dem Kontinent veranla Bt hätte, den Hütten die Verpflichtung aufzuerlegen, ihr Möglichstes zur Zugutemachung oder wenigstens Unschädlichmachung der im Rauchgase enthaltenen Säuren zu tun. Man hat den Ansprüchen der Behörden nach beiden Seiten hin genügt. Ein Teil der Zinkhütten, dem kein Markt für Schwefelsäure offen stand, hat die aus den Flammöfen entweichenden Rauchgase durch Waschen mit dünner Kalkmilch in Türmen von den Säuren befreit, während andere Hütten, die sich in Bezirken befanden, in denen für Schwefelsäure ein Absatz existierte, das Röstgas unvermischt mit Feuergas berzustellen versuchten, um daraus Schwefelsäure zu fabrizieren.

1) R. H a s e n c le ver, Fischers J. B. 1881, 173; E. A. H e r i n g, Die Verdichtung des Hüttenrauches. Stuttgart 1888. 
Unter den zum Zwecke der Blendeabröstung erfunderien Öfen, welche die Herstellung eines für die Schwefelsäurefabrikation geeigneten Gases gestatten, hat sich der mechanische Röstofen von $\mathrm{H}$ e g e l e r als der vollkommenste und geeignetste erwiesen, weil er bei großer Leistung vorzüglich abröstet, auch gleichzeitig ein gutes Röstgas für die Fabrikation von Schwefelsäure erzeugt und in bezug auf Kohleverbrauch und Belegschaft rationeller arbeitet, als die anderen demselben $Z$ wecke dienenden Öfen. Um jedoch das Werden des Ofens, welcher heute einen der vollkommensten metallurgischen Apparate darstellt, verstehen zu können, und um zu ersehen, wie derselbe als Produkt langjähriger Entwicklung, namentlich auch durch Aufnahme der bewährten Bestandteile der Röstöfen anderer Erfinder die heutige Vollkommenheit und kulturelle Bedeutung erlangt hat, ist es nötig, den Stammbaum des Ofens kennen zu lernen. Zu diesem Zwecke muß weit zurückgegriffen werden in die Anfänge der Zinkblendeabröstung, in eine Zeit, als zum ersten Male versucht warde, sie mit der Schwefelsäurefabrikation $\mathrm{zu}$ verknüpfen.

Es ist das bleibende Verdienst der Chemischen Fabrik F. W. Hasenclever \& Cie in Stolberg $^{2}$ ), aus welcher die Rhenania hervorgegangen ist, die Lösung dieser Aufgabe unternommen zu haben. Gleich bei Gründung der Fabrik durch Friedrich Hasenclever, MaxBraun, dem damaligen Direktor der Vieille-Montagne, und dem belgischen Ingenieur $\mathbf{E}$ u g è n e Go di n , im Jahre 1852, war der Plan ins Auge gefaßt worden, die auf den benachbarten Zinkhütten zur Röstung gelangende Zinkblende für die Fabrikation von Schwefelsäure nutzbar zu machen. Vor dem Jahre 1855 wurde sämtliche in den Stolberger Hütten verwendete Blende in gewöhnlichen Flammöfen durch direkte Kohlenfeuerung abgeröstet, wobei die Rauchgase einfach ins Freie ausströmten. In jenem Jahre wurde zum erstenmal die Herstellung von Schwefelsäure versucht. Zwecks Gewinnung eines geeigneten Röstgases ?wurde, vermutlich nach $G$ od in s Angabe, ein kombinierter Flamm- und Muffelofen gebaut, der einen großen Teil des Schwefels zunutze machen sollte und nur einen Teil der sich entwickelnden schwefligen Säure in die freie Luft, den größeren Teil aber in eine Bleikammer abgab, um dort in Schwefelsäure umgewandelt zu werden. Der neue Ofen bestand in seinen wesentlichon Teilen aus einer langen Muffel, unter welcher sich eine Feuerung befand, und welche von den Feuerungsgasen umspült wurde. In die Muffel wurde die Blende aufgegeben, von einem Ende zum anderen gekrählt und schlieBlich im unteren Teile des Ofens mit freier Flamme totgeröstet. Dieser einfache Muffelofen wurde von $G$ o d i n verbessert und seine Idee im Jahre 1865 von $\mathrm{R}$ o be $\mathrm{r} \mathrm{H}$ a s e n c l e ver ausgeführt.

Im G od i n schen Ofen hatte das Erzklein, ehe es auf die von den Feuergasen erhitzte Sohle des Flammofens gelangte, 7 übereinander liegende Muffeln zu passieren. Die unten im Flammofen totgeröstete Blende wurde ausgezogen, die Beschickung der zweiten Platte auf die erste geschoben, die der

2) R. H a s e n c lever, Wiener Weltausstellungsbericht 3, 167; Z. Ver. d. Ing. 1886, 83. dritten auf die zweite usf. und auf die siebente frisches Erz aufgegeben. Die Abröstung erfolgte in diesem Röstofen unter Erzielung reicher Röstgase in befriedigender Weise. Wenngleich der $\mathrm{G}$ o d i n sche Muffelofen einen noch etwas primitiven Apparat darstellt, so muß er doch als der Prototyp aller späteren mehretagigen Röstöfen angeseben werden. G o d i n hat somit das Verdienst, den Bau der ersten mehretagigen Blenderöstöfen veranlaßt zu haben. Wie die Zukunft gezeigt hat, ist dieser Ofen, der mit der bekannten P e r r e t schen Konstruktion $^{3}$ ) viel Ähnlichkeit hatte, verbesserungsfähig gewesen, jedoch $H$ a s e n clever ließ sich bald, nachdem auch für kurze Zeit, 1866-1870, der Gersten höf e r sche Röstofent) zum Blendeabrösten versucht worden war, von dem dem Goding chen Ofen zugrunde liegenden Konstruktionsprinzip, die Etagen horizontal zu bauen, abbringen. Nach $\mathrm{H}$ a s e n cle $\mathrm{v}$ e r s Vorgang hat man seit 1870 ganz allgemein Ófen mit schiefer Ebene, auf der das Erzklein, durch Widerstände gehemmt, in eine Muffel herabrutschte, benutzt. Auch diese von $\mathrm{Hasenclever}$ und $\mathrm{Hel}$ big ${ }^{5}$ ) erfundenen und vielfach verbesserten Ofen gestatten ebensowenig wie der $\mathrm{G}$ o d i n s c h e Etagenofen, die völlige Gewinnung des Schwefels, erfuhren aber durch Hasenclever eine vorzügliche Durchbildung und sind insofern auch von Bedeutung für die Blendeverhüttung geworden, als mittels dieser Öfen die Kunst des Blendeabröstens weiter ausgebildet worden ist.

Die vollständige Lösung der den Zinkhütten vom Staate aufgedrungenen Aufgabe der Beseitigung oder Zugutemachung der schwefligen Säure, die in gewissen Mengen bis dahin immer noch aus den Blenderöstöfen in die freie Luft entströmte, ist jedoch erst K. E i c h h or $n$ und M. L i e bi g ${ }^{8}$ ) in Letmathe in Westfalen gelungen, welchen anfangs der achtziger Jahre zum erstenmal die Verwertung der gesamten bei der Röstung der Blende sich entwickelnden schwefligen Säuro mittels eines neu erfundenen Ofens gelungen ist. Letzterem liegt ebenso, wie dem Etagenofen von $G$ od in, das Prinzip zugrunde, die Röstung sowohl mit äußerer Erhitzung durch fremde Brennstoffe, als auch mit innerer Erhitzung der Muffeln durch die bei der Oxydation des Schwefelzinks entwickelte Wärme zu bewirken, Eichhorn und Liebig gehen jedoch in richtiger Erkenntnis der die vollständige Abröstung bedingenden Umstände, namentlich in dem Bestreben, der Blende äußere Wärme zuzuführen, viel weiter als $G$ od in und vergrößern die Heizfläche in genügendem Maße dadurch, daß sie, nicht wie $\mathrm{G}$ o d i $\mathrm{n}$, nur einem einzigen Herd des mehretagigen Ofens, sondern mehreren äußere Wärme zuführen, und außerdem noch durch Einleiten heißer Oxydationsluft die Wärmezufuhr zwecks Erreichung absoluter Entschwefelung bis zur Vollständigkeit steigern.

3) G. L u n g e, Handb. d. Schwefelsäurefabrikation. 1. Aufl. S. $171-172$.

4) Ibid. 3. Aufl., S. 287.

5) Ibid. S. 310 .

6) Deutsch. Reichspatent Nr. 21032 v. 2./5. 1882; Chem. Industr. 1883, 136 u. 1884, 80; $\mathrm{F}$ is c h e r s Chem. Technologie 1889, 338. 
H a senclever ${ }^{7}$ ) hat später den Ofen von E i c h h or n und $\mathrm{L}$ i e big in einen Doppelofen umgewandelt, indem er zwei solche Öfen mit einer gemeinschaftlichen Mittelwand verband. Als eine Vervollkommnung dieses Doppelofens ist der Ersatz der wenig baltbaren Steinplatten durch flache Gewölbe anzusehen, welche Abänderung die Fortbewegung der Erzpost in der Lïingsachse gestattete und die Standfestigkeit des Ofens in hohem Grade vermehrte. In dieser ebenfalls von $\mathrm{H}$ a s e nc l e ver verbesserten Form hat sich der Röstofen seitdem in der Technik erhalten.

Droi Jabre vor Erfindung des $E$ ic h h or nL i e bigsohen Ofens hat $P$ eter $S$ pe n c e ${ }^{*}$ ) in England, dem $M$ a lé $t r$ a schen Kiesofen, der seit dem Jahre 1872 ziemlich allgemein zur Pyritabröstung eingeführt worden war, größere Dirnensionen gegeben und mit einem mechanischen Rübrwerk versehen. Der Malétraofen wurde bekanntlich mit Rücksicht auf den Handbetrieb nur in verhältnismäßig kleinen Größen gebaut, und man suchte seinerzeit die Leistungsfähigkeit lediglich durch Vervielfältigung zu steigern, indem man Einheit an Einheit reihte und eine größere Anzahl Öfen durch einen gemeinschaftlichen Röstgaskanal verhand. SowohI S p e n c e wie $\mathrm{H}$ e g e I er , letzterer in ganz besonderem Maße, versuchten, die Steigerung der Produktion durch Vergrößerung der Ofeneinheit zu erreichen, welch letztere jedoch die Bewältigung der Rühr- und Bewegungsarbeit nur auf mechanischem Wege, der umgeänderten Bauart des Ofens entsprechend. in der Längsachse, gestattete. Der ursprüngliche Spenceofen ist vieretagig angelegt und als Doppelofen ausgebildet und besitzt am eipen Ofenende als Rührvorrichtung einen auf Schienen maschinell beweglichen Wagen oder ein Gerüst, an dessen senkrechtem Rahmen, der Höho der Etagen entsprechend, Stangen befestigt sind, an deren Ende die sich in den Etagen hin und her bewegenden Krählen angebracht sind.

Nach ähnlichen Grundsätzen, aber unter Berücksichtigung der Eigenart der Blende und der speziellen Bedürfnisse mit dem Zwecke größtmöglicher Leistung bei billigsten Produktionskosten ist der mechanische Blenderöstofen gebaut worden. E d w a r d C. H e g e le r, der Erfinder des Ofens, dessen Konstruktionen seit dem Erlöschen der $\mathrm{Pa}$ tente in den Vereinigten Staaten sowohl den Blendröstöfen wie den Zinköfen beinahe unverändert zugrunde gelegt worden sind, ist ohne $Z$ weifel der bedeutendste Zinkhüttenmann, den die Zinkhüttenindustrie im vorigen Jahrhundert, ja bis heute hervorgebracht hat. Die Matthießen und Hegeler Zinc Co. in $\mathrm{La} \mathrm{Salle}^{9}$ ) ist technisch im wesentlichen seine Schöpfung. Aus kleinsten Anfängen sich entwickelnd, rasch wachsend und aufblühend, später suf breitester Grundlage umgebaut, zeigt dieses große Hüttenwerk in den Teilen wie im Ganzen, in der Baukonstruktion der Ófen und Kammern, wie

7) Handb. d. Schwefelsäurefabrikation v. K. W. $\mathrm{J}$ u r is c h 1893,87

8) Engl. Patent v. 24./12. 1878; deutsch. Reichspatent Nr. 9267; Vereinigt. Staaten Patent Nr. 248521.

9) Die Stadt liegt am Illinoisfluß, im Staate gleichen Namens, die Hütte an den Abhängen des romantischen Little Vermillion. in den maschinellen Ánlagen, in den Verkehrseinrichtungen, wie in der Administration der Hütte, die Hand des großen Ingenieurs und genialen Mechanikers, der grübelnd alle Einzelheiten plant, langsam reifen läßt und schließlich auf nächstem Wege unter Anwendung der einfachsten Mittel zu erreichen sucht, und dessen Werke, trotzdem sie zum Teil natürlicherweise auch das Wesentliche der Errungenschaften anderer Erfinder in sich bergen, doch das Gepräge der Eigenart in hervorragendem Maße besitzen. Als das bedeutendste Werk $\mathrm{H}$ e g e le $\mathrm{r} \mathrm{s}$ ist sein mechanischer Röstofen zu betrachten, den der Erfinder anfangs der achtziger Jahre zu entwickeln begonnen hat. Der Ofen hat im Laufe der Jahre manche Anderung und Verbesserung erfahren; auch sind schließlich seine Größenverhältnisse immer mehr gesteigert worden. Die Patentschrift ${ }^{10}$ ) vom 12./8. 1884 enthält jedoch bereits die wesentlichen Teile des heutigen Ofens. Derselbe ist der Standfestigkeit. und Wärmeökonomie halber, schon von Anfang an, als Doppelofen gedacht, und die Muffeln sind zu beiden Seiten einer Mittelwand in gleicher Höhe angeordnet. Die Herde sind gewölbt, die Muffeln an den beiden Schmalseiten des Ofens offen, während die Langseiten schon mit den zum Einsetzen von Gezähe nötigen Öffnungen versehen sind. Auch der zum Durchziehen der Rechen dienende Mechanismus, welcher späterhin nur noch unwesentliche Veränderungen erlitt, ist schon in der frühesten Konstruktion vorhanden, das Getriebe jedoch nur an einem Ende des Ofens, und zwar in doppelter Ausfïhrung aufgestellt, wodurch angedeutet ist, daß der Erfinder seinerzeit das Durchkrählen des Erzes in ein und derselben Richtung beabsichtigt hat. Die beiden an den Ofenenden stehenden, zur Aufnahme der Rechen dienenden, siebenetagigen Transportwagen mußten nach dem jedesmaligen Durchrechen ausgewechselt werden, zwecks Aufstellung der Rechen im Sinne der Richtung der beabsichtigten Bewegung. Der erste in Patente beschriebene Hegelerofen zeigt daher manches gemeinsame, wenigstens in der Idee, mit dem Spenceschen Pyritofen, und zwar hat $\mathrm{Hegeler}$ in erster Linie den Bewegungsorganismus der Krählen neu ausgestaltet und die Standfestigkeit sowie die Bauart des Ofens in originellerWeise und Einfachheit verbessert. In anderer Hinsicht weicht jedoch der Hegelerofen vom Spenceofen vollständig $a b$, und ist es interessant, daB der Erfinder die zum Abrösten der Blende nötige Wärmezufuhr lediglich mittels stark erbitzter Luft bewirken will. Letztere soll aus zwei unter den Ofenhälften liegenden Kanälen den Röstinuffeln zugeführt werden. Die $\mathrm{Zu}$ leitung geschieht am Ende mittels zweier an beiden Ofenseiten hochgeführten Windkanäle, welche mit den vorhandenen 14 Muffeln durch eine gleiche Zahl von Öffnungen verbunden sind. Die Ableitung der Röstgase erfolgt am entgegengesetzten Ofenende in ähnlicher Weise wie die Zuleitung der heißen Luft, mittels eines am Ofen hochgeführten Abgaskanals.

In einem späteren Stadium der Entwicklung weicht der Ofen von der oben dargestellten Bauart

10) Vereinigtes Staaten Pat. Nr. 303531 v. 12./8. 1884; Wa 1 te r R. I ng a ll s, Metallurgy of Zinc and Cadmium 1903, 146. 
dadurch sehr wesentlich ab, daß die Rührorgane an beiden Enden des Ofens aufgestellt sind, wodurch das Durchkrählen der beiden Ofenhälften wechselseitig und in der heute üblichen Weise erreicht wird.

Später hat sich $\mathrm{H}$ eg el er von der anfangs beabsichtigten Art und Weise der Zufuhr der Wärme, welche lediglich durch erhitzte Luft geschehen sollte, abbringen lassen und hat in eigenartiger Benutzung des dem Ofen von Ei c h h or n und $\mathrm{L}$ i e b i g zu. grunde liegenden Gedankens durch Einlegung von Heizkammern $\mathrm{z}$ wischen die drei untersten Röstmuffeln denselben $Z_{\text {weck }} \mathrm{zu}$ erreichen gesucht. Um den zum Abrösten nötigen heißen Wind zu gewinnen, wurde kalte Luft in einem eisernen Windüberhitzer durch die Verbrennungsabgase der Feuerung vorgewärmt, unter dem Herde der ersten Verbrennungskammer hoch erhitzt und aus mehreren Schlitzen in die erste und zweite Röstmuffel eingeleitet, wo sie, noch weiter erhitzt, sämtlicho Muffeln, eine nach der anderen, durchströmen sollte.

Durch diese Wärmezufuhr durch indirektes Feuer und heißen Wind und durch die Neuanordnung der Krählvorrichtung an beiden Enden des Ofens war im großen und ganzen der Ofen auf seine beutige Höhe erhoben, wenn auch späterhin noch manche Verbesserung an demselben vorgenommen wurde, wie z. B. der Ersatz der Plateauwagen durch Drehgerüste, wodurch der Betrieb eine noch weitere wesentliche Vereinfachung erfahren hat.

Nachdem der Ofen in konstruktiver Hinsicht in allen Teilen ausgearbeitet war und sich in der Folge als ausgezeichneter Röstapparat erwiesen hatte, erfolgto seine weitere Entwicklung in der Richtung der Frhöhung der Röstleistung, welche durch allmähliche Vergrößerung der Dimensionen erreicht wurde. Aus dem 10-Tonnenofen entstand der 20-Tonnenofen: aus letzterem der 40-Tonnenofen, d. h. der Ofen in seiner heutigen Größe.

Der Hegelerofen hat auch späterhin, bis in die Neuzeit hinein, noch Abänderungen erfahren. So hat $\mathrm{Juli}$ u $\mathrm{W}$. He gel e $\mathrm{r}^{11}$ ) den Zugstangen der Getriebe leichtere und sicherere Führung ver. liehen durch Anbringen von Wägelchen am Ende der ersteren und Herstellung einer Eisenbahn wie auch durch Einlegen von Gleitrollen zwischen die Schienen der Bahn. Auch hat man auf anderen Werken, z. B. auf denen der Illinois Zinc Co. in Peru (IIl.) zur Bewegung der Krählschlitten andere Bewegungsorganismen gewählt, indem man die Zug. stangen, stattsie durch Friktionsrollen zu bewegen, an Ketten ohne Ende befestigt, die sich um zwei Rollen drehen. In Anpassung an die Natur des abzuröstenden Erzes hat man an anderen Orten die Zahl der Röstmuffeln und, um der Eigenart des Brennstoffes Rechnung zu tragen, die Heizkammern der Lage und Zahl nach verändert. Auch ist die Art und Weise der Erhitzung der Oxydationsluft und deren Ableitung in die in verschiedenen Höhenlagen sich befindlichen Muffeln zwecks Steigerung oder Verminderung der Luftzufuhr in etwas anderer Weise durchgeführt und verändert worden. Wieder andere Hütten haben die Feuerungsabgase des Röstofens, statt sie in eisernen Windüberhitzern oder $\mathrm{S}$ i e $\mathrm{m}$ e n sschen Wärmespeichern zugute zu machen, unter

11) Vereinigtes Staaten Patent Nr. 592006 v. 19./10. 1897 .
Dampfkesseln zur Dampf- oder Krafterzeugung benutzt. Bei diesen Abänderungen handelt es sich jedoch in den wenigsten Fällen um eigentliche Verbesserungen, sondern vielmehr um Rücksichtnahme auf jeweilige Verhältnisse, hervorgerufen durch die geographische Lage der Hütten, durch die zur Verfügung stehenden Baufonds, durch Anpassung an den Brennstoff, wie z. B. beim Heizen mit Naturgas, oder an den persönlichen Geschmack der Verwaltung usw., so daß man sagen kann, daß der von $\mathrm{E}$. C. $\mathrm{H}$ e g e $\mathrm{l}$ e $\mathrm{r}$ erfundene und ausgestaltete Röstofen späterhin keine eigentliche Verbesserung mehr erfahren und in der Gestalt und Konstruktion, die ihm der Erfinder verlieh, den Höhepunkt der Entwicklung und inneren Durchbildung erreicht hat.

Hand in Hand mit der qualitativen und quantitativen Ausbildung der Röstanlage ging diejenige der Schwefelsäurekammerapparatur. Die Gloverund Gay-Lussactürme, die Staubkammern, Säure. hebevorrichtungen usw. wurden konstruktiv verbessert, in den Abmessungen den Verhältnissen entsprechend verändert und wirksamer ausgestaltet. Insbesondere war es das Bedürfnis, die Bewegung der Oxydationsluft im Röstofen kontrollieren, nach Wunsch variieren und gleichmäßig gestalten zu können, ferner das Bestreben, die den Gasstrom hemmenden Widerstände, wie sie die Füllungen der Turmanlagen darboten, überwinden zu können, welche $\mathrm{He}$ g e le r zur Einführung des Ventilators ${ }^{12}$ ) in den. Kammerbetrieb bewogen haben. Einerseits führte diese wichtige und einschneidende Neuerung zur besseren Abröstung der Blende, andererseits zur Produktionserhöhung und zur intensiveren Ausnutzung von Röstofen und Kammer. Die Leistungen $\mathrm{H}$ e $\mathrm{g}$ e $\mathrm{l}$ e $\mathrm{r}$ s auf diesem Gebiete sind dem. nach nicht nur von spezieller Bedeutung für die Blendeverhüttung, weil mittels der neuen Röstapparatur dio Technik des Blenderöstens in hohem Grade vervollkommnet worden ist, sondern von ganz allgemeiner. Bedeutung für die chemische Industrie überhaupt geworden, weil auch die Schwefelsäurefabrikation neue mächtige Anregungen zur Vervollkommnung und Weiterentwicklung erhalten hat.

In Europa benutzt heute die Mehrzahl der Schwefelsäure fabrizierenden Zinkhütten den $\mathrm{E}$ i c h horn-Liebig ofen in der ihm von $\mathrm{H}$ as enclever gegebenen Form, ausnahmsweise auch den Brownofen $^{13}$ ) zum Abrösten der Blende und zur Herstellung der Röstgase, welche zur Fabrikation von Schwefelsäure dienen, weil in diesem Lande da, wo Zinkhütten sind, auch ein Bedarf für Schwefelsäure vorhanden ist, und die Hütten zur Herstellung derselben durch gesetzliche Vorschriften veranlaßt worden sind. In den Vereinigten Staaten dagegen, wo bis jetzt Vorschriften dieser Art nicht in Kraft sind, und ein Markt für Schwefelsäure bäufig nicht vorbanden ist, läßt man in der überwiegenden ZahI der Fälle die Röstgase unbenutzt in die Luft entweichen. Auch verwendet man in Nordamerika der besonderen Arbeiter- und Lohnverhältnisse halber,

12) Vgl. O. M ü h l h äus er, diese Z. 16, 672

13) Metallurgy of Zinc and Cadmium by W. R. Ing a l Is , 138. 
an Stelle der älteren direkt geheizten Handröstöfen öfters wie in Kansas mit Naturgas geheizte, mechanische Röstöfen von mehr oder weniger großer Leistungsfähigkeit, die nacb den Erfindern als Brown-14), Ropp ${ }^{15}$ ). und Zellwegeröfen ${ }^{16}$ ) bezeichnet werden. In diesen automatischen Fortschaufelungsöfen wird die Erzpost mittels Krählwagen meohanisch fortbewegt. Uberall jedoch, wo ein Absatz für Schwefelsäure existiert oder ein solcher geschaffen werden kann, benutzt man drüben nach dem Vorgang der Matthiessen \& Hegeler Zino Co. seit anfang dieses Jahrhunderts ganz allgemein den Hegelerofen, wie dann derselbe in nicht oder wenig veränderter Form auf den nachstehenden Zinkhütten in einem oder mehreren Exemplaren erbaut worden ist. Auch in Europa und Australien ist die Einführung des Hegelerofens nunmehr in Betracht gezogen.

1. Matthiessen \& Hegeler Zinc Co., La Salle, Ill. . . . . . . . . 1884-1896

2. Mlinois Zinc Co. Peru, Dl. . . . 1900

3. Standard Acid Co., Jola, Kansas 1902

4. United States Zinc Co., Pueblo, Co. 1903

5. Graselli Chemical Co., Cleveland, O. 1904

6. United Zinc and Chemical Co., Argentine, K. . . . . . . . . . . 1904

7. Mineral Point Zinc Co. Mineral Point, Wis. . . . . . . . . .

8. Hegeler Bros., Danville, Ill. . . .
In La Salle, Peru, Jola, Argentine, Danville und Cleveland werden die Röstgase in Kammersystemen verarbeitet, in Mineral Point und Depue dagegen mittels des Schröder-Grillo-Kontaktverfahrens zur Herstellung von Monohydrat und Oleum verwendet. Nur die United States Zinc Co. in Pueblo, welche zurzeit für Schwefelsäure nooh keinen Absatz hat, verwendet den Hegelerofen, der geringeren Zinkverflüchtigung halber, an Stelle der direkt geheizten mechanischen Brown-, Ropp- oder Zellwegeröfen.

Den Stammbaum des Hegelerofens und seine Verbreitung habe ich in folgendem Schema zur Darstellung $\mathrm{zu}$ bringen versucht.

\section{Über die Methylalkoholvergiftung.} Von Dr. med. R. MüLleR.

(Nach einem vor Chemikern gehaltenen Vortrage.)

(Eingeg. 19./1, 1910.)

Während die Wirkung des Âthylalkohols allgemein bekannt ist, ist der Einfluß des Methylalkohols, der als Bestandteil der genossenen spirituösen Getränke für gewöhnlich kaum in Betracht kommt, auf den menschlichen und tierischen Körper weniger bekannt. Trotzdem sind schon zahlreiche Vergiftungen mit Methylalkohol vorgekommen, dadurch, daBdieser zur Verfälschung alko holischer Getränke benutzt wurde, ferner dadurch, daß er in Amerika in Patentmedizinen und Essenzen statt Äthylalkohol verwandt warde. So wurde er in Ingweressenz von Jamaica, in Pfefferminzessenz und in Bayrum festgestellt, und schlieB. lich in der Technik als Zusatz zu Harzlösungen und Polituren. Es ist da. her, bei der Bedeutung, welche die Vergiftung mit Methylalkohol hat, interessant, näher auf dieselbe einzugehen.

Was zunächst die akute Vergiftung mit Methylalkohol betrifft, so gibt $\mathrm{Poh} \mathbf{l}^{1}$ ) auf Grund des Tierversuches eine Darstellung von ihr, aus der bereits das für sie Charakteristische hervorgeht. P O h I schreibt: „Reicht man einem kräftigen Hund Methylalkohol in genügend großer Dosis und passender Verdünnung, etwa $40-50 \mathrm{ccm}$ Alkohol für einen 8-10 kg schweren Hund, so bemerkt man wie beim Athylalkohol nach einiger Zeit das Auf* treten von Koordinationsstörungen, die sich $\mathrm{zu}$ nächst unter zunehmendem Bewegungstrieb bis zum Taumeln steigern; dann wird das Tier schlafsüshtig und verfällt in stundenlang andauernden

1) Pohl, Uber die Oxydation des Methylund Athylalkohols im Tierkörper. Schmiedebergs Archiv 31, 281 ff. (1893). 